silver stains technique. Had this been done, it is possible that appropriate treatment could have been instituted to have prevented death from disseminated histoplasmosis, even though treatment is admittedly not without dangers.

\section{The Interpretation of Respiratory Tract Histology in Cot Deaths}

E. TAPP, D. M. JONES, AND J. O'H. TOBIN (Withington Hospital, Manchester) The diagnosis of 'cot death' is essentially one of exclusion, and is made in sudden unexpected deaths in infancy where all recognized causes of death have been eliminated. The main problem, therefore, in the respiratory tract is to determine whether a particular lesion is significant enough to cause death.

With this in mind, the histological degree of inflammation in the respiratory tract of 1939 sudden unexpected deaths has been assessed and has been correlated with the bacteria isolated from them. The type of bacteria isolated appears to give some indication as to the significance of degrees of inflammation in the tracheobronchial tree which on histological grounds alone would have been doubtful.

Virus isolations from the lungs have been low $(16 \%)$, but were found particularly in cases which histologically showed acute bronchiolitis. Viruses were also isolated from two cases showing only minor degrees of inflammation in the lung parenchyma. In addition immunoflorescent studies of specific antibody to respiratory virus in the $\operatorname{Ig}_{\mathrm{a}}, \mathrm{Ig}_{\mathrm{g}}$, and $\mathrm{Ig}_{\mathrm{m}}$ fractions of the serum indicate evidence of recent virus infection in five other cases with only minor histological lesions in the lung parenchyma. The significance of these findings is discussed.

Filing and Retrieval of ' $2 \times 2$ ' Transparencies in Morbid Anatomy

R. A. G. BROWN (Ninewells Hospital, Dundee) An inexpensive and reliable indexing, filing, and retrieval system for ' $2 \times 2$ ' transparencies within a department of pathology is described. Indexing is based upon an abridgement of the Systematized Nomenclature of Pathology and retrieval upon punched feature cards. Using plastic slide wallets holding 24 slides, 2400 transparencies and the associated index cards can be conveniently stored in one drawer of a standard filing cabinet.
Endoscopic Biopsy and the Diagnosis of Duodenitis

T. J. BETTERIDGE (RAF Institute of Pathology, Halton) The advent of endoscopy has imposed further burdens upon the pathologist in the assessment of changes and their significance. The problem of limits of confidence is discussed with respect to the diagnosis of duodenitis.

A simple classification is described and related to the diagnosis of 200 duodenal endoscopy biopsies. The classification relates to cellular and architectural changes. It is not felt that gastric metaplasia is of value in the grading of duodenitis.

Classification may be of limited value when set against justified clinical bias in the choice of biopsy site, but a logical assessment with a standardized format is of value to pathologists and clinicians in maintaining a consistent standard of evaluation.

\section{Histopathology Reports and Medical} Records-A Matter of Chance?

I. M. MAGRATH (Orpington, Kent) Most histopathology departments suffer from telephone enquiries about reports. The reading of reports over the telephone, and their receipt, by non-medical staff is a practice officially frowned on but, in fact, tolerated as inevitable. While some impatience by clinicians with the speed of decalcification or of special stains in difficult cases is understandable, requests for results already sent out need examination.

Some 500 reports each from two laboratories with different speeds and methods of receiving and processing specimens and of delivering reports were followed in an attempt to determine factors which might cause long-term failure of reports to reach their destination. It seemed likely that specimens which had reached the laboratory poorly identified, with incomplete or incorrect details, would be at risk in proportion to these inadequacies. In fact, no such consistent reason could be found for the nucleus of missing reports, and it became clear that many poorly or wrongly detailed reports reached the case notes because there existed duplications and mechanisms which ensured this, whether by accident or design; the fallibility of some elaborate and expensive devices used in records systems was also shown.
While the causes of disappearance $\stackrel{0}{\overrightarrow{0}}$ some reports, and the quantitative valie of the covering mechanisms for many the remainder could hardly be assesset, it seemed desirable that any attempt कo 'rationalize' procedures in a laboratoogy office, secretariat, or hospital recores department should be preceded by Фa careful study of the local circumstancess for each hospital, as such streamlinifrpg might cause a serious drop in the arrika of reports.

\section{An Epidemic Due to Serratia marcesceino in a Neurosurgical Unit}

K. B. ROGERS AND B. GITTENS (Childrends Hospital, Birmingham) Over a 10-mond period the operation sites of 16 of $1 \$ 1$ patients who had major intracrangl operations developed infections due to Serratia marcescens.

Until the characteristic colonial appeiance of serratia on MacConkey's medium was appreciated, it was thought that to of the first four infections were caused Enterobacter liquefaciens.

The infection never followed sinal operations or operations on Thurs $\overrightarrow{y s}$, when the anaesthetist did not meâfife central arterial blood pressures. This le to a realization that two months befof the first infection became manifest thepe had been a modification of the apparatis with which the central arterial blood pressure was measured. Serratia wब्बुs isolated from a tube that was no longer required, but which had been inadverte再ly left in place and was being connected to the new recording system.

The serratia in this epidemic hado relatively low virulence. The infection became manifest in nine patients betwe 99 and 266 days after operation and ordy six patients showed evidence of infectiষ̋ as early as $\mathbf{1 2}$ days after operation; atso in five patients serratia was recovered $\frac{2}{t}$ the end of operations from the skfin incision before it was sutured and nome developed infections. This is in marked contrast to the fulminating septicaenfia with thrombocytopenia, purpura, afy hyperpyrexia some years previous amongst the infants, in a small epidemif, who became infected with serratia through the incision made to give int venous fluids.

Antibodies to serratia were found in the sera of the infected patients and the possible value of this finding is discusset. 